\title{
A Model of the Zonally Averaged Stratification and Overturning in the Southern Ocean
}

\author{
DIRK OLBERS \\ Alfred Wegener Institute for Polar and Marine Research, Bremerhaven, Germany \\ MARTIN VISBECK* \\ Lamont-Doherty Earth Observatory, Columbia University, Palisades, New York
}

(Manuscript received 26 January 2004, in final form 21 December 2004)

\begin{abstract}
The ocean area south of the Antarctic Circumpolar Current (ACC) frontal system is a region of major watermass modification. Influx of North Atlantic Deep Water (NADW), small-scale mixing, eddy transport and diffusion, as well as the fluxes of momentum and buoyancy at the sea surface combine in a complex array of processes to generate the unique stratification of the Southern Ocean with its southward uprising isopycnals and northward flux of Antarctic Intermediate Water (AAIW) and Antarctic Bottom Water. Comprehensive analytical models of this scenario are rare. The authors develop and apply a model based on zonally and temporally averaged theory to explain the conversion of NADW into AAIW with all of the aforementioned processes contained in an extremely simplified way. Eddies appear via a transformed Eulerian mean (TEM) approach with a conventional downgradient parameterization of the meridional density flux. The structure of the eddy coefficient is estimated from hydrographic and wind stress data by a simple inverse approach. Mixing is limited to a near-surface layer and is treated in a most simple entrainment form. The model determines the zonal mean density stratification in the Southern Ocean and the baroclinic transport of the ACC from the applied wind stress and the surface density flux and unravels the role and importance of the different processes responsible for shaping the stratification (Ekman and eddy-induced advection and pumping, mixing, surface buoyancy flux, and eddy-induced diffusion). All of these processes must be present to yield an agreement between the simulated stratification and the observed one, but details of their parameterization might not be too critical. The ACC transport is shown to have a contribution forced by the local wind stress as well as another contribution relating to the nonlocal forcing by wind stress and density flux over the entire Antarctic zone.
\end{abstract}

\section{Introduction}

Much of the perception of the Southern Ocean is centered on the zonal circulation around Antarctica in the Antarctic Circumpolar Current system (ACC). The meridional structure of the stratification has long been used to infer the zonal transport of the ACC [for recent analysis see Rintoul and Sokolov (2001) and Cunning-

\footnotetext{
* Current affiliation: Leibniz-Institute for Marine Sciences (IFM-GEOMAR), Kiel, Germany.
}

Corresponding author address: Dirk Olbers, Alfred Wegener Institute for Polar and Marine Research, Bussestrasse 24, 27570 Bremerhaven, Germany.

E-mail: dolbers@awi-bremerhaven.de ham et al. (2003)], and observational evidence of water mass storage and spreading have lead, as early as 1933 , to the classical view of the meridional overturning and ventilation of water masses (see, e.g., Sverdrup et al. 1942). A linkage of the meridional overturning and the zonal circulation in a dynamical perspective is only beginning to emerge (e.g., Marshall 1997; Speer et al. 2000; Marshall and Radko 2003). What has been found in the recent decade or so for the dynamical balance of the zonal momentum of the ACC-an overwhelming importance of mesoscale eddies (see the reviews of Rintoul et al. 2001 and Olbers et al. 2004 and the literature cited there) - is also true for the overturning: eddies must accomplish a major task in shaping and balancing the meridional circulation (see Fig. 1). This is not surprising because the balance equations for the zonal momentum and the meridional transport profile 


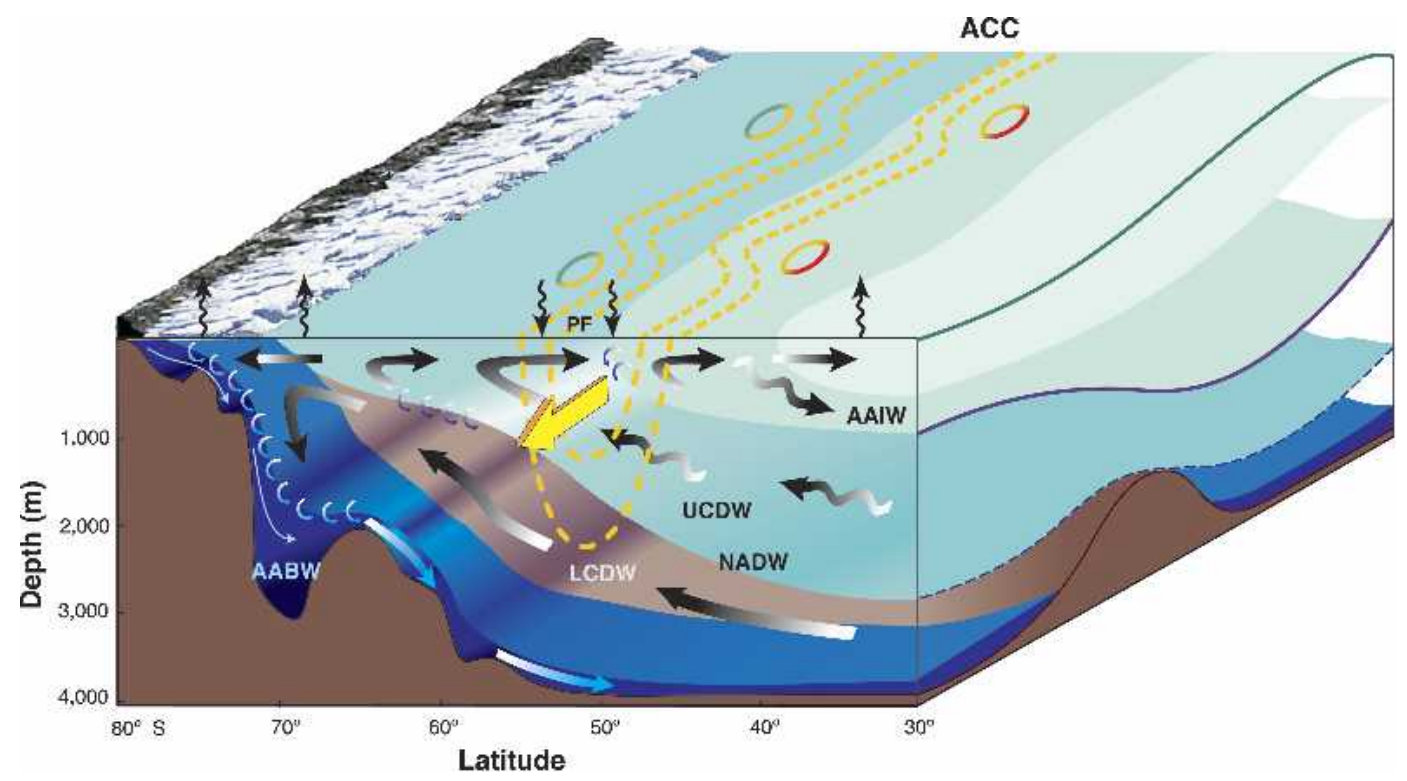

FIG. 1. A sketch of the ACC system showing the zonal flow and the meridional overturning circulation and water masses. Antarctica is at the left side. The east-west section displays the isopycnal and sea surface tilts in relation to submarine ridges, which are necessary to sustain the bottom form stress signatures (see Olbers et al. 2004). The curly arrows at the surface indicate the buoyancy flux; the arrows attached to the isopycnals represent turbulent mixing. An upper cell is formed primarily by northward Ekman transport beneath the strong westerly winds and southward transport in the Upper Circumpolar Deep Water (UCDW) layer. The lower cell is driven primarily by formation of dense AABW near the Antarctic continent and inflowing Lower Circumpolar Deep Water (UCDW or NADW). Redrawn using a figure from Speer et al. (2000).

are identical. But the involvement of eddies and the implied problem of handling them in analytical (and numerical) models may have been the hampering factor leading to the quite late development of theories of Southern Ocean overturning.

Marshall (1997) discusses the process of subduction by eddies of newly formed water masses from the mixed layer and presents a zonal mean model of the Southern Ocean overturning. He uses an isopycnal framework. We follow and extend the concept presented in Marshall and Radko (2003), which is based on Eulerian equations but has the same physical contents. It is assumed that all mixing and watermass formation processes take place in an upper layer of the oceanbasically a turbulent mixed layer where Ekman transport and pumping is established by the wind and eddies, and buoyancy is imprinted on the surface waters by heat and freshwater flux from the overlying atmosphere. The ocean interior is assumed void of turbulence, but eddies are present that transport and mix substances along isopycnals. We refer to this concept as "adiabatic eddy regime" and see it as an extreme scenario. The real ocean might have substantial mixing by turbulence in the interior as well, in particular close to the bottom (see, e.g., Heywood et al. 2002; Naveira Garabato et al. 2004). Eddies might contribute also in the ocean interior by a diapycnal flux to watermass formation as well.

We extend Marshall and Radko's concept to a prognostic theory that predicts the zonal-mean density field entirely from wind stress and surface buoyancy flux data [a National Centers for Environmental Prediction (NCEP) reanalysis dataset is used; see section 3]. The model is based on a quasi-streamline mean (we use an average following the ACC path) and temporal-mean transformed Eulerian mean (TEM) theory (see, e.g., Andrews et al. 1987; Olbers and Ivchenko 2001) outlined in section 2 and is separated into an interior adiabatic eddy regime where the density and momentum balances are solved by a characteristics approach (see sections 4 and 7) and an upper layer with basically mixed layer properties, with an attached "slope layer" in which the vertically completely mixed state blends to the interior isopycnals with finite slopes (see section 6). Turbulent mixing is limited to this upper-layer stack. Eddy transport of density is parameterized by a conventional downgradient form with a specified lateral eddy diffusivity. Its depth dependence and meridional dependence are estimated from hydrographic data [the Special Analysis Center (SAC) analysis is used (available online at http://www.bsh.de/en/Marine\%20data/ Observations/Climate/WOCEAIMS/WGHC/index 
.jsp)] in section 5. A summary and conclusion are found in section 8 .

\section{Zonally averaged equations and parameterizations}

For steady conditions the quasi-streamline averaged conservation equations for potential density $\rho$ and vertically integrated momentum (from the surface to depth z) are (see, e.g., Olbers and Ivchenko 2001)

$$
\begin{aligned}
\mathcal{I}(\psi, B) & =-\frac{\partial}{\partial y} \overline{v^{\prime} \rho^{\prime}}-\frac{\partial}{\partial z} \overline{w^{\prime} \rho^{\prime}}-\frac{\partial J}{\partial z} \text { and } \\
-f \psi & =\tau_{0}-\tau+\mathcal{F} .
\end{aligned}
$$

Integration is assumed along a path following the mean ACC (such as mean SSH or another index of the pathway) so that standing eddies become small and may be neglected, and $y$ is the cross-stream coordinate. The overbar denotes a time average over eddy time scales and a zonal average, $B$ is the zonal and time average of the potential density $\rho, \psi$ is the streamfunction of the mean Eulerian flow ( $\psi$ is transport per unit length of the circumpolar path, $\left.\bar{v}=-\psi_{z}, \bar{w}=\psi_{y}\right), \tau_{0}$ is the zonal wind stress, $\tau$ is the subgrid-scale (SGS) stress, and $J$ is the vertical SGS (diapycnal) flux of $\rho$ (the meridional component is omitted in view of the larger eddy fluxes). Lateral SGS and Reynolds stresses of momentum are neglected. Last, $\mathcal{F}(y, z)$ is the bottom form stress exerted by the depth range from the top the hills down to the respective level $z$; thus $\mathcal{F}=0$ for depths in the unblocked water column. Boundary conditions are $\tau(z$ $=0)=\tau_{0}, J(z=0)=-\mathcal{H}_{0}$ (the surface density flux $\mathcal{H}_{0}$ results from heat and freshwater exchange with the atmosphere), and the usual zero flux conditions for momentum and density at the bottom $z=-h(y)$ (this is the maximum depth on each path $y=$ const).

To utilize the equations we need to specify the SGS fluxes $J$ and $\tau$, the eddy-induced flux vector $\mathbf{F}=\left(\overline{v^{\prime} \rho^{\prime}}\right.$, $\left.\overline{w^{\prime} \rho^{\prime}}\right)$, and the bottom form stress $\mathcal{F}$. We follow the most simple concepts. The interior ocean (below an upperocean layer of depth $z=-a$ ) is assumed adiabatic with respect to SGS mixing; that is, $J \equiv 0$. The density flux $J$ will appear only as mixing terms at interfaces in the upper ocean and will there be parameterized in an entrainment fashion. The stress $\tau$ is written as a body force, which is nonzero only in the surface Ekman layer (above a depth $z=-d$, embedded in the upper-ocean layer; i.e., $d<a$ ). Hence $\tau=E(z) \tau_{0}$ where $E(z)=1$ at the top and zero below $z=-d$. In the deep-water column the form stress becomes effective. Its amplitude is constrained by the vertically integrated balance of momentum: since the Eulerian mass transport stream-

\section{stress structure function $\mathrm{T}(\mathrm{z})$}

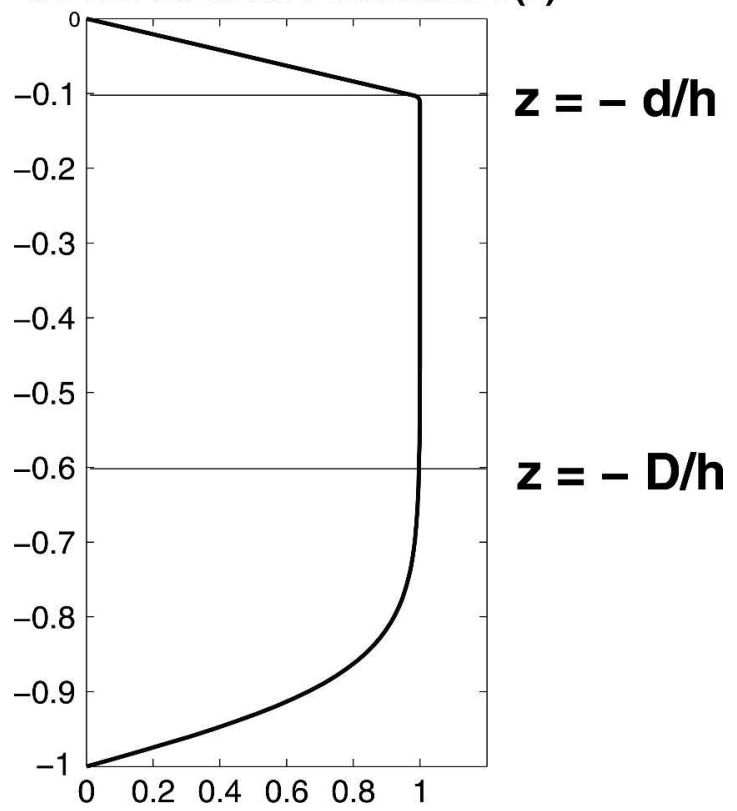

FIG. 2. Sketch of the structure function $T(z)$ as function of $z / h$. The depth $z=-d$ marks the base of the upper frictional boundary layer; $z=-h$ is the bottom (maximum depth along circumpolar path), and below $z=-D$ the water column is blocked by topography.

function $\psi$ vanishes on the surface and the bottom $z=$ $-h$, we find from (1) the balance $\tau_{0}+\mathcal{F}(-h)=0$, which represents the balance of the zonal momentum of the ACC. Thus we put $\mathcal{F}(z)=-\tau_{0} F(z)$ with $F(z)$ increasing from zero at the hill top, say $z=-D$, to unity at the maximum depth $z=-h$. Then, the momentum balance implies

$$
\psi=T(z) \mathcal{M}(y)
$$

with $T(z)=1-E(z)-F(z)$ and $\mathcal{M}=-\tau_{0} / f($ Ekman transport). ${ }^{1}$ The structure function $T(z)$ of the winddriven circulation must come at a later stage from a dynamical theory. Here we prescribe it as sketched in Fig. 2. Notice that $T(z)$ must be differentiable.

It has become customary to use the transformed Eulerian mean (see, e.g., Andrews et al. 1987; McIntosh and McDougall 1996) formulation as framework of the zonal averaged problem (1). The aim of TEM is to account for the common belief that eddies transport properties mainly along isopycnals. The formulations of TEM presented below all express the eddy flux vector as

$$
\mathbf{F}=\left(\overline{v^{\prime} \rho^{\prime}}, \overline{w^{\prime} \rho^{\prime}}\right)=-\chi\left(-B_{z}, B_{y}\right)+\boldsymbol{\varphi} .
$$

\footnotetext{
${ }^{1}$ In general, transport refers to transport per unit circumpolar length.
} 
Apparently, $\chi$ is a streamfunction because $\boldsymbol{\nabla} \cdot \mathbf{F}=$ $-\mathcal{I}(\chi, B)+\boldsymbol{\nabla} \cdot \boldsymbol{\varphi}$. The splitting of $\mathbf{F}$ separates out part of the eddy flux to act as advection velocity for mean density. With the two relations (3), any choice of one of the three components $\chi, \varphi^{y}, \varphi^{z}$ determines the other two in terms of $\mathbf{F}$. As the $\chi$ part is tangential to isopycnals, the canonical choice is to have $\varphi$ normal to isopycnals; that is, $\varphi=-\kappa \nabla B$, which implies

$\chi=\frac{\overline{v^{\prime} \rho^{\prime}} B_{z}-\overline{w^{\prime} \rho^{\prime}} B_{y}}{|\nabla B|^{2}}$ and $\kappa=-\frac{\overline{v^{\prime} \rho^{\prime}} B_{y}+\overline{w^{\prime} \rho^{\prime}} B_{z}}{|\nabla B|^{2}}$.

Apparently, $\kappa$ is a diapycnal diffusivity. This obvious choice was proposed by Andrews and McIntyre (1978). Other suggestions are from Andrews and McIntyre (1976): $\varphi=\left(0, \varphi^{z}\right)$ resulting in $\chi=\overline{v^{\prime} \rho^{\prime}} / B_{z}, \varphi^{z}=-s \overline{v^{\prime} \rho^{\prime}}$ $+\overline{w^{\prime} \rho^{\prime}}$; and Held and Schneider (1999): $\boldsymbol{\varphi}=\left(\varphi^{y}, 0\right)$ resulting in $\chi=-\overline{w^{\prime} \rho^{\prime}} / B_{y}, \varphi^{y}=\overline{v^{\prime} \rho^{\prime}}-\overline{w^{\prime} \rho^{\prime}} / s$. Here $s=$ $-B_{y} / B_{z}$ is the slope of the zonal and time mean isopycnals ( $s=d z / d y$ on $B=$ const). In any case, the density balance becomes

$$
\mathcal{I}\left(\psi_{\mathrm{res}}, B\right)=-\frac{\partial J}{\partial z}-\nabla \cdot \boldsymbol{\varphi}
$$

where the total advection is represented by $\psi_{\text {res }}=\psi+$ $\chi$, which is the streamfunction of the residual circulation. Note that no approximation has been made; (5) is identical to the original density balance in (1). Neglect of the remaining divergence on the rhs leads to the approximation of the eddy-adiabatic regime: assuming F to be oriented along isopycnals we find that the $\chi$ in the three different choices are identical and $\kappa=\varphi \equiv 0$. We assume this adiabatic condition with no eddy mixing across mean isopycnals to be valid in the ocean interior (below $z=-a$ ).

At all depths however, we assume a downgradient form for the lateral eddy flux (e.g., Green 1970; Stone 1972),

$$
\overline{v^{\prime} \rho^{\prime}}=-K \frac{\partial B}{\partial y}
$$

with some still to be specified $(y, z)$ dependence for the lateral eddy diffusivity $K$. In the interior where $\mathbf{F}$ is along isopycnals we have $\overline{w^{\prime} \rho^{\prime}}=s \overline{v^{\prime} \rho^{\prime}}$. Then (6) implies a parameterization of the vertical flux as well, $\overline{w^{\prime} \rho^{\prime}}=$ $-K s B_{y}=K s^{2} B_{z}$, which is up the vertical density gradient: for ACC conditions where $B_{y}<0$ (and $B_{z}<0$ in general) the slope $s$ is negative and, thus, the eddy flux vector $\mathbf{F}$ is pointing downward along isopycnals. In this adiabatic eddy regime we then find $\chi=K s$, which is the Gent-McWilliams parameterization of the eddy streamfunction (Gent and McWilliams 1990), hence

$$
\psi_{\text {res }}=\psi+\chi=T(z) \mathcal{M}(y)+K s
$$

In the upper-ocean layer, above $z=-a$, these relations do not apply, particularly not if the density gets vertically mixed and thus $s$ becomes infinite. We propose a simple parameterization of $\overline{w^{\prime} \rho^{\prime}}$ for this part of the water column further below in section 6 .

\section{The averaged SAC climatology and NCEP forcing data}

We have made use of two datasets to analyze the climatological mean ocean state and its exchanges of heat, freshwater, and momentum with the atmosphere. The first is a compilation and gridding of a large number of oceanographic measurements known as the World Ocean Circulation Experiment (WOCE) hydrographic SAC climatology. From that we have constructed the pathway of the ACC by finding the latitude of the $3^{\circ} \mathrm{C}$ isotherm at a depth of $200 \mathrm{~m}$. Applying some smoothing we find the path shown in Fig. 3 .

We then shifted all meridional sections by their departure from the zonally averaged ACC position. Last, all data were sorted along their new "latitude circles" and averaged to obtain sections of potential density, temperature, and salinity (see the quasi-streamline averaged salinity in Fig. 3 as example). In this alongstream average only the middle $50 \%$ of the sorted data were used to avoid contamination by outliers. We obtain the well-known mean stratification of the Southern Ocean with southward and upward sloping isopycnals and the dominating AAIW signature in the salinity field. The magnitude of the isopycnal slope decreases from $-1.5 \times 10^{-3}$ to less than $-0.5 \times 10^{-3}$ between about 1000-m depth and the upper layers (see Fig. 4).

To develop our model of the mean density structure we need an estimate of the surface momentum, heat, and freshwater fluxes. Our estimates are based on the adjusted National Center for Atmospheric Research (NCAR)-National Centers for Environmental Prediction reanalysis data. Wind stress data were obtained by averaging along mean ACC streamlines and converted to a surface Ekman transport and wind-stress-driven overturning Euler streamfunction using Eq. (2) (see Fig. 5). The maximum Ekman transport is about $30 \mathrm{~Sv}$ $\left(\mathrm{Sv} \equiv 10^{6} \mathrm{~m}^{3} \mathrm{~s}^{-1}\right)$, occurring at $-51^{\circ}$ latitude with upwelling to the south and downwelling to the north of this latitude. Surface buoyancy loss depends on the combined air-sea heat and freshwater flux, which were both obtained from the NCEP-NCAR dataset. The zonally averaged net heat loss seemed biased and was reduced everywhere by $10 \mathrm{~W} \mathrm{~m}^{-2}$. Consistent with other estimates we find oceanic heat gain of about $10 \mathrm{~W}$ 

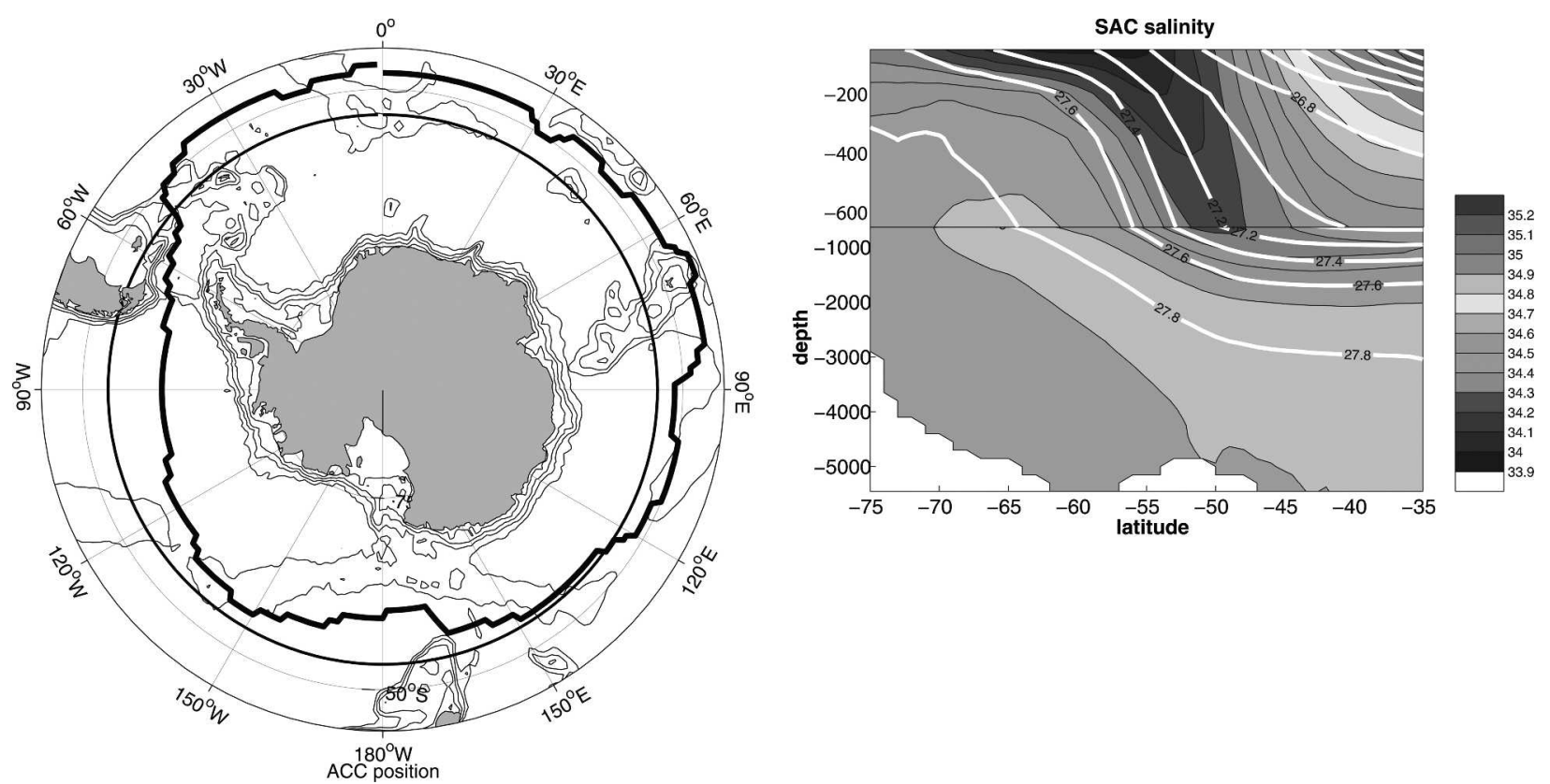

FIG. 3. (left) Path of the ACC using the $3^{\circ} \mathrm{C}$ isotherm at $200-\mathrm{m}$ depth as criterion. (right) Averaged potential density (white contours) on top of the salinity. The downward-sloping salinity tongue is associated with the density range of AAIW (note that latitudes refer here to the average latitude in the path-averaging approach). Computed from the SAC climatology.

$\mathrm{m}^{-2}$ over the core of the ACC and cooling of a similar magnitude north and south of the stream (see Fig. 5). Our adjusted heat loss estimate is consistent with the implied poleward ocean heat transport calculations by Trenberth and Caron (2001) and an extensive air-sea flux forcing comparison study by Fritzsch et al. (2000). The freshwater flux at the surface shows a net freshening south of $-50^{\circ}$ of about $1.5 \times 10^{-5} \mathrm{~kg} \mathrm{~m}^{-2} \mathrm{~s}^{-1}$ and about a similar amount of net evaporation around $-30^{\circ}$, causing a significant modification of the surface density. Only between $-47^{\circ}$ and $-37^{\circ}$ is the freshwater contribution to the density flux smaller than the air-sea heat flux. Our final estimate of air-sea density flux yields a reduction of the surface density south of $-47^{\circ}$ and an increase of about $0.6 \times 10^{-5} \mathrm{~kg} \mathrm{~m}^{-2} \mathrm{~s}^{-1}$ north of $-40^{\circ}$ (see Fig. 5). However, the expected error on the surface density flux is rather large and could be as much as $100 \%$.
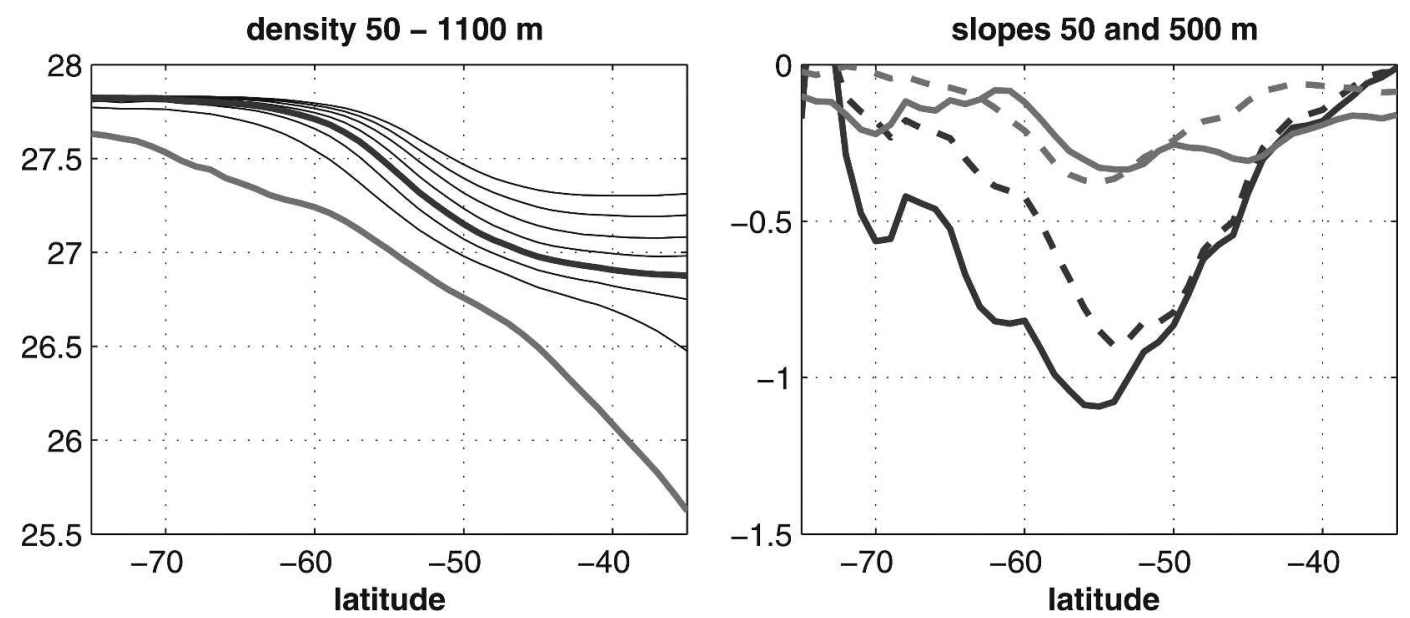

FIG. 4. (left) Density in the upper eight levels of the SAC climatology: 50- and 500-m levels are fat lines. (right) Slopes $\left(\times 10^{3}\right)$ at these levels [full line: local slope in the SAC grid; dashed: slope from Eq. (11)]. 

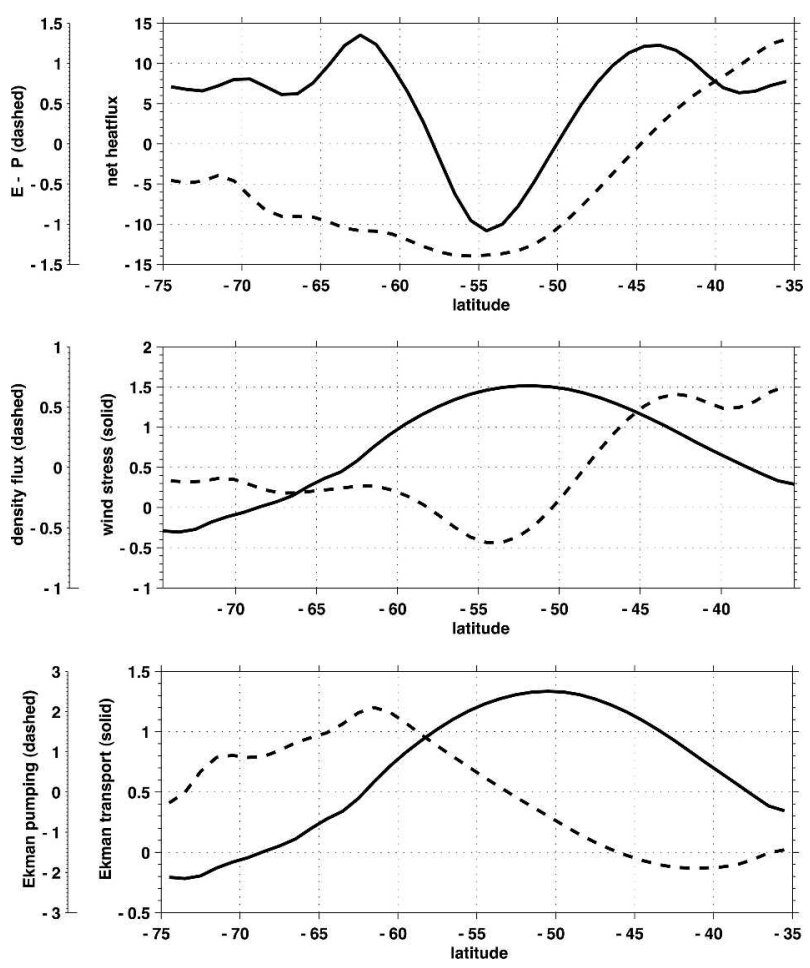

FIG. 5. The forcing data obtained by quasi-streamline averaging from the NCEP analysis (units $10^{-5} \mathrm{~s}^{-1}$ for $E-P, \mathrm{~W} \mathrm{~m}^{-2}$ for heat flux, $10^{-4} \mathrm{~m}^{2} \mathrm{~s}^{-2}$ for wind stress, $10^{-6} \mathrm{~kg} \mathrm{~m}^{-2} \mathrm{~s}^{-1}$ for density flux, $10^{-6} \mathrm{~m} \mathrm{~s}^{-1}$ for Ekman pumping, and $\mathrm{m}^{2} \mathrm{~s}^{-1}$ for Ekman transport). Notice that the buoyancy flux in the most southern part of Fig. 1 does not coincide with our estimate of surface density flux. The dashed curves correspond to the left scale in each panel; the full curves correspond to the right scale.

\section{The adiabatic eddy regime}

In the adiabatic regime we have $\mathcal{I}\left(\psi_{\mathrm{res}}, B\right)=0$. An integral is easily found: isolines of $\psi_{\mathrm{res}}$ and $B$ must coincide in this state, thus

$$
\psi_{\mathrm{res}}=K s+T \mathcal{M}=\Pi(B),
$$

with a unknown function $\Pi(B)$. By physical reasoning this function is determined by inflow conditions into the interior domain where the residual circulation advects boundary values of density inside. Interior points in the adiabatic regime, which are not directly connected to the inflow boundary cannot be handled by (8). Given the functional relation $\Pi(B),(8)$ is differential equation for the $B$ field, which is readily solved as it is a characteristics differential problem of quasi-linear form. Equivalently, we can solve the adiabatic density balance, conveniently written as a differential equation for the slope,

$$
\frac{\partial}{\partial y}(K s+T \mathcal{M})+s \frac{\partial}{\partial z} K s=0 .
$$

Characteristics of this equation have the slope $s$ everywhere; they are indeed the isopycnals. Initial data are required on some nonisopycnal curve; in our case this will be the depth level $z=-a$. For a vertically constant eddy diffusivity an analytical solution is given in the appendix.

\section{Constraints on eddy diffusivity}

In the adiabatic regime the residual circulation is set by the imbalance between the wind stress and eddydriven overturning. In addition, the residual streamfunction must be constant on isopycnal surfaces, as given by (8). Here we explore the dependence of the eddy diffusivity $K$ on rates of AAIW formation and upwelling NADW. We loosely define AAIW as all the waters with a density lighter than $27.68 \mathrm{~kg} \mathrm{~m}^{-3}$ and denser water as NADW (see Fig. 3). An analytical function $\Pi(B)$ was then constructed by merging two Gaussians with a maximum at the interface density of 27.68 $\mathrm{kg} \mathrm{m}^{-3}$ and a width of $0.5 \mathrm{~kg} \mathrm{~m}^{-3}$ for the AAIW side and only $0.09 \mathrm{~kg} \mathrm{~m}^{-3}$ for NADW (displayed in the upper panel of Fig. 6). The amplitudes of the two functions were adjusted to reflect the desired AAIW and NADW transports. Now a zonally averaged $K$ can be computed by (8), given $\Pi(B)$ from Fig. 6 , the observed density $\left(\sigma_{0}\right)$ slopes from the SAC climatology (Fig. 4), and $\mathcal{M}$ computed from the zonally averaged wind stress using the NCEP-NCAR reanalysis data (Fig. 5).

Whereas our knowledge of watermass properties and stratification in the different basins of the Southern Ocean is supported by an enormous number of publications (see, e.g., the review in Rintoul et al. 2001), there are only a few attempts to quantify the transport rates, either basin wise or as a total zonal integral. Schmitz (1995, 1996a,b) combined a large number of published estimates for the individual branches of the overturning circulation and suggests that about $53 \mathrm{~Sv}$ of southward-flowing NADW and UCDW are converted to northward-flowing $6 \mathrm{~Sv}$ of intermediate (IW) and thermocline waters (TW) and $48 \mathrm{~Sv}$ of bottom water (AABW and LCDW). These numbers are very similar to the inverse model results of Sloyan and Rintoul (2001). The transport rates for the density classes of LCDW, UCDW, and the intermediate waters are much different in the individual basins and even show different directions. Thus, in Sloyan and Rintoul's model, 10 Sv of UCDW and $7 \mathrm{~Sv}$ of LCDW enter the Southern Ocean from the Atlantic Ocean, while $45 \mathrm{~Sv}$ of UCDW enter and $31 \mathrm{~Sv}$ of LCDW leave in the Pacific and Indian Ocean basins, respectively. The diversity for IW and TW is similar: in the Atlantic $10 \mathrm{~Sv}$ of TW/IW leave, in the Indian $14 \mathrm{~Sv}$ enter, and in the Pacific $8 \mathrm{~Sv}$ 

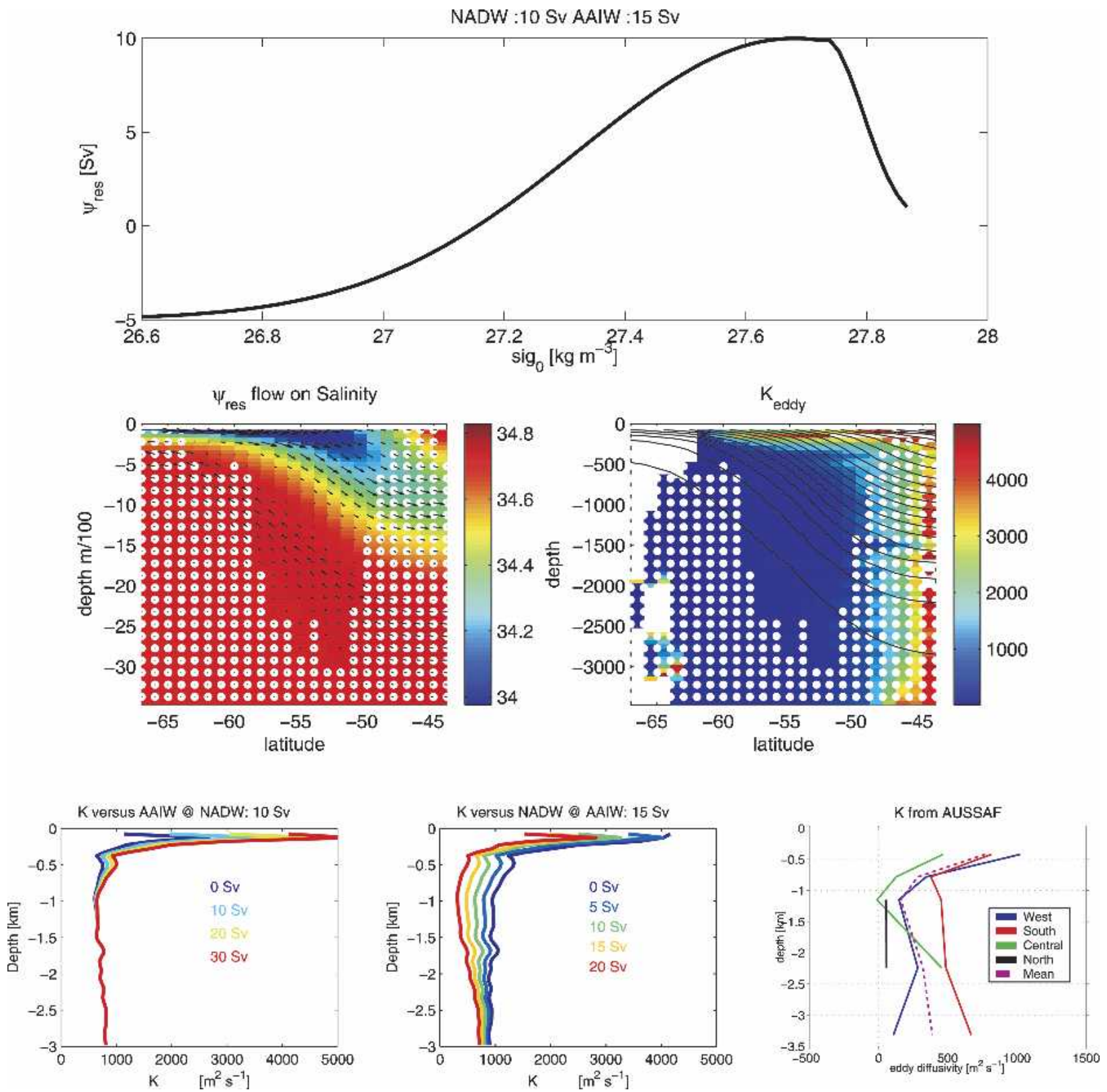

FIG. 6. (top) Plot of $\Pi(B)$ constructed from a pair of Gaussian functions with an AAIW formation rate of 15 Sv and NADW transport of $10 \mathrm{~Sv}$. (middle left) Residual circulation (arrows) overplotted on salinity; (middle right) $K$ with isopycnals overplotted. The white dots represent regions where the free flow is blocked by land more than $4 \%$ of the way. (bottom left) Vertical profiles of horizontally averaged $K$ for different $\Pi(B)$ with AAIW formation rates varying between 0 and $30 \mathrm{~Sv}$ and a fixed NADW transport of $10 \mathrm{~Sv}$. (bottom middle) Same with NADW formation rates varying between 0 and $20 \mathrm{~Sv}$ and a fixed AAIW transport of 15 Sv. (bottom right) The diffusivity determined from the four moorings of the AUSSAF array (H. Phillips 2004, personal communication).

leave the Southern Ocean at about $30^{\circ}-40^{\circ} \mathrm{S}$. For the most part, these estimates from direct observations or inverse calculation appear larger than transport rates inferred from passive tracers. Thus, on the basis of chlorofluorocarbon observations, Orsi et al. (2001, 2002) suggests a meridional circulation with $8.1 \mathrm{~Sv}$ of AABW, 21.1 of southward LCDW, and 3.6 of northward
UCDW. The great difference between the circulation schemes based on direct transport calculations and those indirectly inferred from tracers must be seen in an eddy-induced circulation, which finds its manifestation in the tracer field but is not considered in geostrophic calculations.

We take $15 \mathrm{~Sv}$ of northward intermediate-water 
transport and $10 \mathrm{~Sv}$ of southward deep-water transport as a reasonable choice of $\Pi(B)$ (but will vary these rates in the following analysis). Figure 6 shows the required local eddy diffusivity $K$ in the middle-right panel. In general it increases toward the north and upward in the water column with values near $800 \mathrm{~m}^{2} \mathrm{~s}^{-1}$ in the deep ocean, increasing to more than $3000 \mathrm{~m}^{2} \mathrm{~s}^{-1}$ at $200-\mathrm{m}$ depth. Here one might argue that the ocean is not anymore strictly adiabatic.

This framework allows us to readily explore the sensitivity of the water mass formation rates to different choices of $K$ or vice versa. Figure 6 (lower-left panel) shows that AAIW transport depends mainly on $K$ in the upper $800 \mathrm{~m}$. At $200-\mathrm{m}$ depth $K$ increases from about $2000 \mathrm{~m}^{2} \mathrm{~s}^{-1}$ for zero AAIW formation to above $4000 \mathrm{~m}^{2} \mathrm{~s}^{-1}$ required for $20 \mathrm{~Sv}$ of AAIW transport. The NADW transport increases with decreasing $K$ (see Fig. 6 lower-middle panel) and the deep ocean $K$ values vary only between 800 and $1000 \mathrm{~m}^{2} \mathrm{~s}^{-1}$ for moderate NADW transports. However, for large NADW transports of more that $10 \mathrm{~Sv}$, the required intermediate values are significantly lower than in the deep ocean. We suspect that this is not consistent with baroclinic eddies dynamics.

Our results are broadly consistent with findings from an array of moorings in the ACC south of Australia (Phillips and Rintoul 2000), which show a significant increase in eddy heat flux and eddy kinetic energy above $2000 \mathrm{~m}$ to about $400-\mathrm{m}$ depth. The eddy diffusivity at the four moorings is shown in Fig. 6 (lowerright panel; H. Phillips 2004, personal communication). It is calculated using the daily rotating, currentfollowing reference frame. The time series of velocity and temperature are then bandpassed ( $40 \mathrm{~h}$ to 90 days) to remove frequencies outside the eddy band. The mean cross-stream temperature gradient near the mooring sites is calculated from the SR3 cruises. The four estimates of the diffusivity reveal a similar depth dependence as our findings, being constant with values of a few hundred meters squared per second and increasing toward shallower depths. We would expect further increase at even shallower depths that were not sampled during this campaign.

\section{The upper diabatic layer}

In the density balance of the upper diabatic layer, $-a$ $\leq z \leq 0$, the diabatic SGS and eddy terms must be retained. We cannot provide analytical solutions as for the interior ocean and therefore take a different approach by keeping all terms in the balance but considering integrated budgets, thus largely avoiding a determination of details of the vertical density structure. A simple density structure,

$$
\begin{aligned}
& B(y, z)=B_{d}(y) \text { for } 0 \leq z \leq-d \text { and } \\
& B(y, z)=B_{d}(y) \frac{z+a}{a-d}-B_{a}(y) \frac{z+d}{a-d} \\
& \text { for }-d \leq z \leq-a,
\end{aligned}
$$

with two $y$-dependent densities, $B_{d}(y)$ and $B_{a}(y)$, will be implemented in the budget equations derived below. This structure represents a mixed layer of depth $d$ on top of a slope layer with vertically constant density gradient $B_{z}=\left(B_{d}-B_{a}\right) /(a-d)$. The slope in the slope layer is, however, not constant in the vertical. The slopes at the interfaces are

$$
\begin{aligned}
& s_{d}=s(y, z=-d)=\frac{\partial B_{d}}{\partial y} \frac{a-d}{B_{a}-B_{d}} \text { and } \\
& s_{a}=s(y, z=-a)=\frac{\partial B_{a}}{\partial y} \frac{a-d}{B_{a}-B_{d}} .
\end{aligned}
$$

The slope layer is the starting point to the internal characteristics as outlined above.

We use the complete density balance equation in the form (1) to derive budget equations for the mixed layer and the slope layer. Integration over these layers yields

$$
\mathcal{M} \frac{\partial B_{d}}{\partial y}-\left.\overline{w^{\prime} \rho^{\prime}}\right|_{-d}=\mathcal{H}_{0}-\mathcal{H}_{d}+\frac{\partial}{\partial y} \mathcal{K}_{m} \frac{\partial B_{d}}{\partial y}
$$

and

$$
\begin{aligned}
\left(B_{d}-B_{a}\right) \frac{\partial \mathcal{M}}{\partial y}+\left.\overline{w^{\prime} \rho^{\prime}}\right|_{-a} ^{-d}= & \mathcal{H}_{d}-\mathcal{H}_{a}+\frac{\partial}{\partial y} \mathcal{K}_{d} \frac{\partial B_{d}}{\partial y} \\
& +\frac{\partial}{\partial y} \mathcal{K}_{a} \frac{\partial B_{a}}{\partial y} .
\end{aligned}
$$

The structure functions (10) have been inserted into the diffusion terms and three weighted (integrated) diffusivities have been defined:

$$
\begin{aligned}
\mathcal{K}_{m} & =\int_{-d}^{0} K d z, \quad \mathcal{K}_{d}=\int_{-a}^{-d} K \frac{z+a}{a-d} d z, \text { and } \\
\mathcal{K}_{a} & =-\int_{-a}^{-d} K \frac{z+d}{a-d} d z,
\end{aligned}
$$

which are still functions of $y$. Notice that they are positive if $K$ is positive. The diffusivities are computed in further applications from a simple profile: $K=K_{s}$ is vertically constant in the mixed layer and drops linearly in the slope layer to an interior value $K_{i}$ at $z=-a$ (cf. with Fig. 6). This yields $\mathcal{K}_{m}=d K_{s}, \mathcal{K}_{d}=(a-d)\left(K_{s} / 3\right.$ $\left.+K_{i} / 6\right)$, and $\mathcal{K}_{a}=(a-d)\left(K_{s} / 6+K_{i} / 3\right)$.

The quantities $\mathcal{H}_{0}, \mathcal{H}_{d}$, and $\mathcal{H}_{a}$ are the fluxes of density across the respective interfaces. Mixing by subgrid processes will be treated simply:

$$
\mathcal{H}_{d}=\alpha_{d}\left(B_{d}-B_{a}\right) \text { and } \mathcal{H}_{a}=\alpha_{a}\left(B_{a}-\tilde{B}\right),
$$


where $\alpha_{d}$ and $\alpha_{a}$ characterize the mixing efficiency. The "deep" density $\tilde{B}$ is mixed in from a certain range below the slope layer. In the stand-alone simulations of the upper-layer equations considered below it will be taken from observed SAC climatology. The complete coupling of the upper layers to the interior requires determining $\tilde{B}$ consistently from the boundary conditions (see section 7). The fluid mixed in at the base of the slope layer has the density $\tilde{B}$. But at the mixed layer base $z=-d$ it is not $B_{a}$ [as one may falsely infer from (14)] but rather an integral of the density profile around $z=-d$ over a mixing length $\Delta$, which is mixed with the mixed layer density $B_{d}$. We take $\alpha_{d}=1 / 2 \alpha_{a} \Delta^{2} /[(a-d)(d$ $+\Delta)$ ], which is generally much smaller than $\alpha_{a}$.

Evidently, the above Eqs. (12) could be used to determine $B_{d}(y)$ and $B_{a}(y)$ from the flux $\mathcal{H}_{0}(y)$ and the Ekman transport $\mathscr{M}(y)$ (prescribed as given in Fig. 5). A remaining task is to adequately parameterize the vertical eddy flux $\overline{w^{\prime} \rho^{\prime}}$ (or the Held-Schneider eddy streamfunction $\left.\psi^{*}=-\overline{w^{\prime} \rho^{\prime}} / B_{y}\right)$ at the mixed layer base and the slope-layer base as well. Since the diapycnal diffusivity $\kappa$ equals the lateral eddy diffusivity $K$ for $B_{z}=0$ (mixed layer) and $\kappa \rightarrow 0$, approaching the adiabatic interior, it seems reasonable to express $\kappa$ as $\kappa=K G(s)$ where $G(s)$ should be small for $|s| \ll s_{c}$ while $G(s) \rightarrow 1$ for $|s| \gg s_{c}$. The critical slope $s_{c}$ must be larger than typical slopes in the interior regime $\left(s_{c} \gg 10^{-3}\right)$. We thus set

$$
\overline{w^{\prime} \rho^{\prime}}=-\psi^{*} B_{y}=-K B_{y} W(s) .
$$

The previous adiabatic eddy case is indeed recovered for $W=s$. To extend this form to large and even infinite slopes we require that the flux $\overline{w^{\prime} \rho^{\prime}}$ becomes arrested if the slope exceeds some critical value. We take

$$
W(s)=s\left\{\begin{array}{ccc}
1 & \text { for } & |s| \leq s_{c} \\
s_{c}|s| & \text { for } & |s| \geq s_{c}
\end{array} .\right.
$$

It becomes obvious that for this flux arresting parameterization (16) the eddy transport streamfunction becomes limited as well; however, at rather large values $|s|$ $\sim 1$ of the slope, and only for these large slopes, the diffusivity $\kappa$ approaches $K$. It should be mentioned that in our applications the arresting property of the above parameterization only came to work during iteration and grid refinement of the integration routines. In the final solution the slopes are generally below the critical value.

We finally write the upper-layer equations in the form

$$
\begin{aligned}
\left(\mathcal{K}_{m} B_{d}^{\prime}\right)^{\prime} & =-\mathcal{H}_{0}+\left(\mathcal{M}+\psi_{d}^{*}\right) B_{d}^{\prime}-\alpha_{d}\left(B_{a}-B_{d}\right) \quad \text { and } \\
\left(\mathcal{K}_{a} B_{a}^{\prime}\right)^{\prime}+\left(\mathcal{K}_{d} B_{d}^{\prime}\right)^{\prime} & =\left(\mathcal{M}+\psi_{a}^{*}\right)^{\prime}\left(B_{a}-\tilde{B}_{a}\right)-\mathcal{M}^{\prime}\left(B_{a}-B_{d}\right)+\psi_{a}^{*} B_{a}^{\prime}-\psi_{d}^{*} B_{d}^{\prime}+\alpha_{d}\left(B_{a}-B_{d}\right)+\alpha_{a}\left(B_{a}-\tilde{B}\right) .
\end{aligned}
$$

The $y$ derivative is denoted for simplicity by a dash and the eddy transport streamfunction is $\psi^{*}=K W(s)$. We have augmented the balance with upstream conditions for the vertical pumping $\left(\mathcal{M}+\psi_{a}^{*}\right)^{\prime}$ at $z=-a$. Hence, $\tilde{B}_{a}$ is the upstream value of density at $z=-a$ with $\tilde{B}_{a}$ $=B_{a}$ in case of downward pumping. There is no such term at the mixed base since we have assumed continuous stratification at $z=-d$.

We like to draw attention to a specific property of these balances: communication with the interior ocean does only occur by mixing at the slope-layer base, that is, only if $\alpha_{a} \neq 0$, and by the upstream term, which is effective in case of upwelling, that is, $\left(\mathcal{M}+\psi_{a}^{*}\right)^{\prime}>0$. If mixing at the slope-layer base is not operating (i.e., $\alpha_{a}$ $=0$ ), only diffusion and eddy advection can prevent complete mixing $\left(B_{d}=B_{a}\right)$ down to the slope-layer base in downwelling regions. We get for $B_{a} \rightarrow B_{d}$ an infinite slope, $s_{a}=s_{d} \rightarrow \pm \infty$, so that the structure function $W$ goes to the arrested state. If $K$ does not change in the slope layer, we obviously get $\psi_{a}^{*} B_{a}^{\prime}-\psi_{d}^{*} B_{d}^{\prime} \rightarrow$ $\pm K s_{c}\left(B_{a}-B_{d}\right)^{\prime}$. We thus summarize: It is essentially mixing at the slope-layer base and diffusion that can prevent the slope layer from reaching a well-mixed condition in case of downwelling.

The mixed layer balance with absent mixing at the base,

$$
\left(\mathcal{M}+\psi_{d}^{*}\right) B_{d}^{\prime}=\mathcal{H}_{0}+\left(\mathcal{K}_{m} B_{d}^{\prime}\right)^{\prime},
$$

was used by Marshall and Radko (2003) in a diagnostic model of the overturning. They prescribe the density $B_{d}$ and the flux $\mathcal{H}_{0}$ by simple analytical structure functions and estimate the streamfunction $\mathcal{M}+\psi_{d}^{*}$ at the mixed layer base. Figure 7 displays the result for our forcing data (climatological data given by Fig. 5) and the SAC density. The diffusive term is small for reasonable diffusivities; it is therefore omitted. There is upward pumping by wind and eddies of roughly $10 \mathrm{~Sv}$ between $-70^{\circ}$ and $-53.6^{\circ}$ and downwelling to the north of roughly $40 \mathrm{~Sv}$. The switch occurs at the critical density $B_{d}=26.95$. When compared with the AAIW signal shown in Fig. 3, this is not a realistic scenario of the overturning in the Southern Ocean. In Marshall and Radko's application, who use simple synthetic data for 

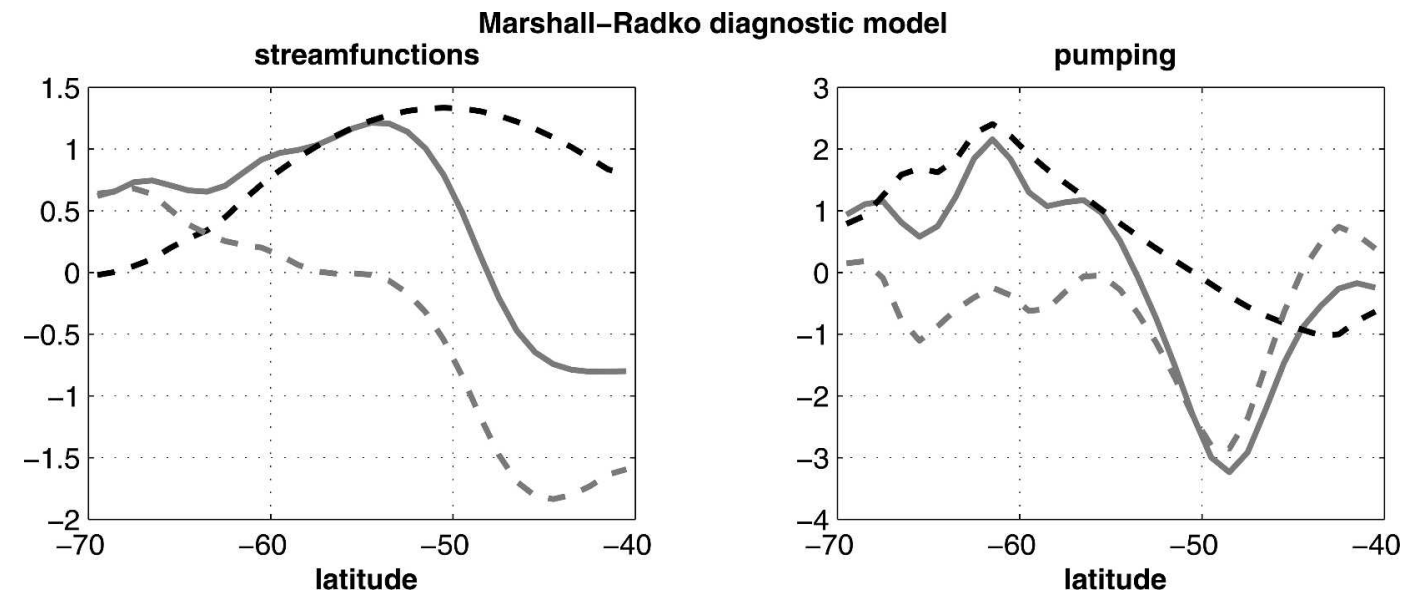

FIG. 7. Marshall-Radko advective solution: (left) streamfunctions and (right) pumping velocities (upper dashed curves: Ekman; lower dashed curves: eddy; full curves: total). Units are $\mathrm{m}^{2} \mathrm{~s}^{-1}$ and $10^{-6} \mathrm{~m} \mathrm{~s}^{-1}$, respectively.

$\mathcal{H}_{0}$ and $B_{y}$, the diagnostic solution looks more promising. We conclude that either our forcing or density data is not consistent or that the neglect of mixing at the interface $z=-d$ leads to these errors.

We integrate the full model Eqs. (17) for the upper layers $^{2}$ (with Dirichlet boundary conditions at both ends and $\tilde{B}$ taken from SAC density data at $800-\mathrm{m}$ depth). The results are exemplified in Fig. 8. The parameter values (given in the figure caption) produce a reasonable fit to the observations (with both forcing functions considered, upper row of the figure), both for the upper-layer densities and the slopes. The good simulation-in comparison with the SAC climatology data - of the densities and the slopes is remarkable. We should like to point out that the chosen parameter values are reasonable (the $\alpha$ values correspond to a vertical diffusivity $K_{v}=d \alpha \sim 3 \times 10^{-4} \mathrm{~m}^{2} \mathrm{~s}^{-1}$ ) but, nevertheless, the solution is quite strongly dependent on them.

Changes by a factor of 2 in either of the $K$ or $\alpha$ deform the density profiles and consequently the slopes significantly. Here it must be remembered that the interior density field will be constructed entirely from the slope at the slope-layer base and that slight changes might result in profoundly different characteristics and interior density structure (see next section). In Fig. 9 we demonstrate the model's performance for modified parameter sets. All of these simulations are less perfect in comparison with the SAC data than the one in Fig. 8

\footnotetext{
${ }^{2}$ In all solutions to come, the forcing functions are polynomial approximation of the wind stress and density flux data shown in Fig. 5. The numerical integrations are performed with equations in spherical coordinates, using MATLAB routines ode45 and bvp4c. We continue to use Cartesian expressions in the text.
}

and details differ quite strongly. There is, however, a quite robust feature in all of them: the alternating pattern of ups and downs in the vertical pumping (by Ekman and eddies), in particular the switch from upward pumping south of about $-60^{\circ} \mathrm{S}$ to downward pumping around $-55^{\circ} \mathrm{S}$ and again upward motion to the north of $-50^{\circ} \mathrm{S}$. This pattern is produced by the surface density flux (see Fig. 10 presenting a case with zero density flux) and by the eddies, acting mostly against the pumping induced by the wind field. We interpret this feature as the upcoming NADW, the downward motion of the AAIW, and upward motion of mode water. In the standard case shown in the upper row of Fig. 8 the pumping of AAIW has a rate of $3 \mathrm{~Sv}$, the NADW has $13 \mathrm{~Sv}$.

\section{The interior density field and zonal transport}

The interior density field is constructed from the slope at the slope-layer base and results from integration of the characteristic equation (9). The solutions for the upper layer shown in Fig. 8 yields the characteristics displayed in Fig. 10. They represent both the upperlayer solution and the interior density in form of the characteristics. The upper two panels refer to the standard case with both forcing functions active. The left upper panel shows the resulting a depth profile of the density field at the northern boundary of the integration domain. The ability of the model to simulate the climatological density state is surely convincing. A better agreement cannot be expected (and should not be attempted by further parameter tuning) since the forcing data from NCEP have a profound level of uncertainty (see section 3) and need not at all be completely consistent with the climatological density from SAC. 

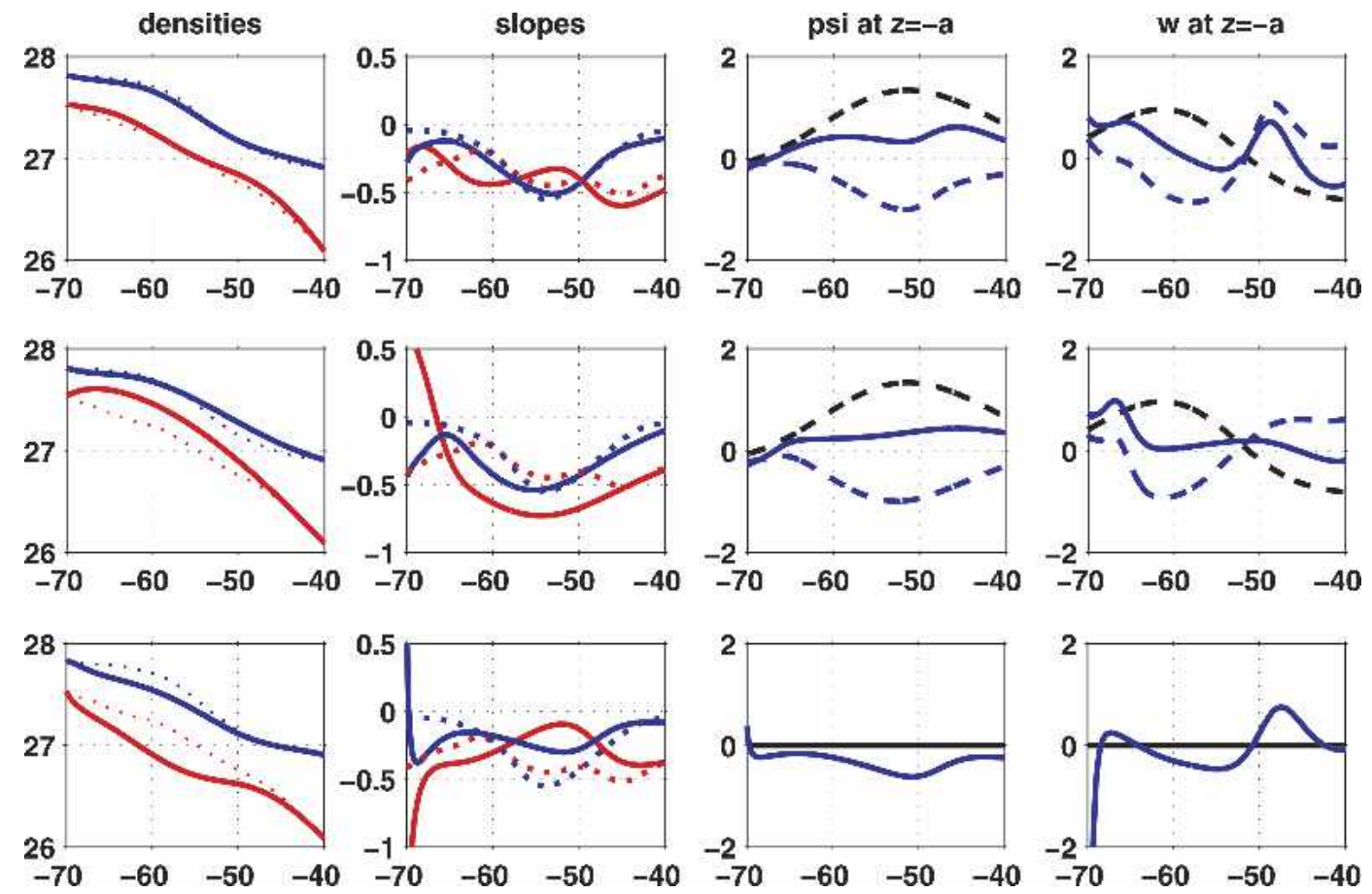

FIG. 8. Solutions for parameter values $K_{s}=1000 \mathrm{~m}^{2} \mathrm{~s}^{-1}, K_{i}=500 \mathrm{~m}^{2} \mathrm{~s}^{-1}$ at the southern boundary, both linearly increasing by a factor of 5 toward the northern side, $\alpha_{d}=8.44 \times 10^{-7} \mathrm{~m} \mathrm{~s}^{-1}, \alpha_{a}=3 \times 10^{-6} \mathrm{~m} \mathrm{~s}^{-1}, d=100 \mathrm{~m}, a$ $=500 \mathrm{~m}$. (top) Forcing by wind stress and density flux. Using the same parameters as above, (middle) the forcing by density flux is switched off or (bottom) the wind stress is switched off. In each row, the left two panels display the resulting density fields $B_{d}$ (red) and $B_{a}$ (blue) and slopes; they are compared with the SAC climatology (dotted). The right two panels show the streamfunctions and pumping velocities at the slope-layer base $z=$ $-a$ : Ekman streamfunction (black dashed), eddy streamfunction (blue dashed), and residual streamfunction (blue full).

The form of the interior characteristics heavily depend on details of the spatial structure of the eddy diffusivity $K(y, z)$. Slight deformations of the $y$ or $z$ dependencies of $K$ directly affect the local slopes and then propagate further into the interior. For the simulation shown in Fig. 10 we have used the most simple form oriented at the diffusivity estimates discussed in section 5: $K(y, z)$ is vertically constant and a linear function of $y$ with a significant increase toward the north that yields an almost perfect reproduction of the AAIW densities (left panel of Fig. 10) at the northern boundary. Surely, the characteristics that result from the alternative parameter choices in Fig. 9 are different.

The lower row of Fig. 10 displays the interior solutions for zero density flux (left panel) and for zero wind stress forcing (right panel), respectively. Mixing and eddy parameters are identical to the previous simulation. The densities and slopes in the upper layer do not fit the observations as well (see Fig. 8), as is the case with both forcing functions included. Neglect of density flux (shown in the lower-left panel of Fig. 10) wipes out the downwelling AAIW signal almost completely whereas it is reaching to too-great depths in an overexaggerated fashion in the case of zero wind stress (lowerright panel of Fig. 10). In the latter simulation the northward Ekman advection is seen clearly missing in the upper layer, leading to an extremely shallow thermocline.

The interior solution allows one to compute the baroclinic zonal transport of the ACC using the thermal wind balance $U_{z}=(g / f) B_{y}$ where $U$ is the zonal velocity (averaged in a quasi-streamline way). Since the interior density field is completely specified by conditions at the slope-layer interface and by the Ekman pumping velocity and the interior eddy diffusivity (see section 4), the density gradient $B_{y}$ at an interior depth $z$ can be mapped onto the gradient $B_{a}^{\prime}$ at that interface (see the appendix), resulting in

$$
U_{z}(y, z)=\frac{g}{f} \frac{d y^{\dagger}}{d y} B_{a}^{\prime}\left(y^{\dagger}\right),
$$

where $y^{\dagger}$ is the footprint on $z=-a$ of the isopycnal that passes the point $(y, z)$ in the interior. The shear $U_{z}$ at the latitude $y$ and depth level $z$ is thus associated with 

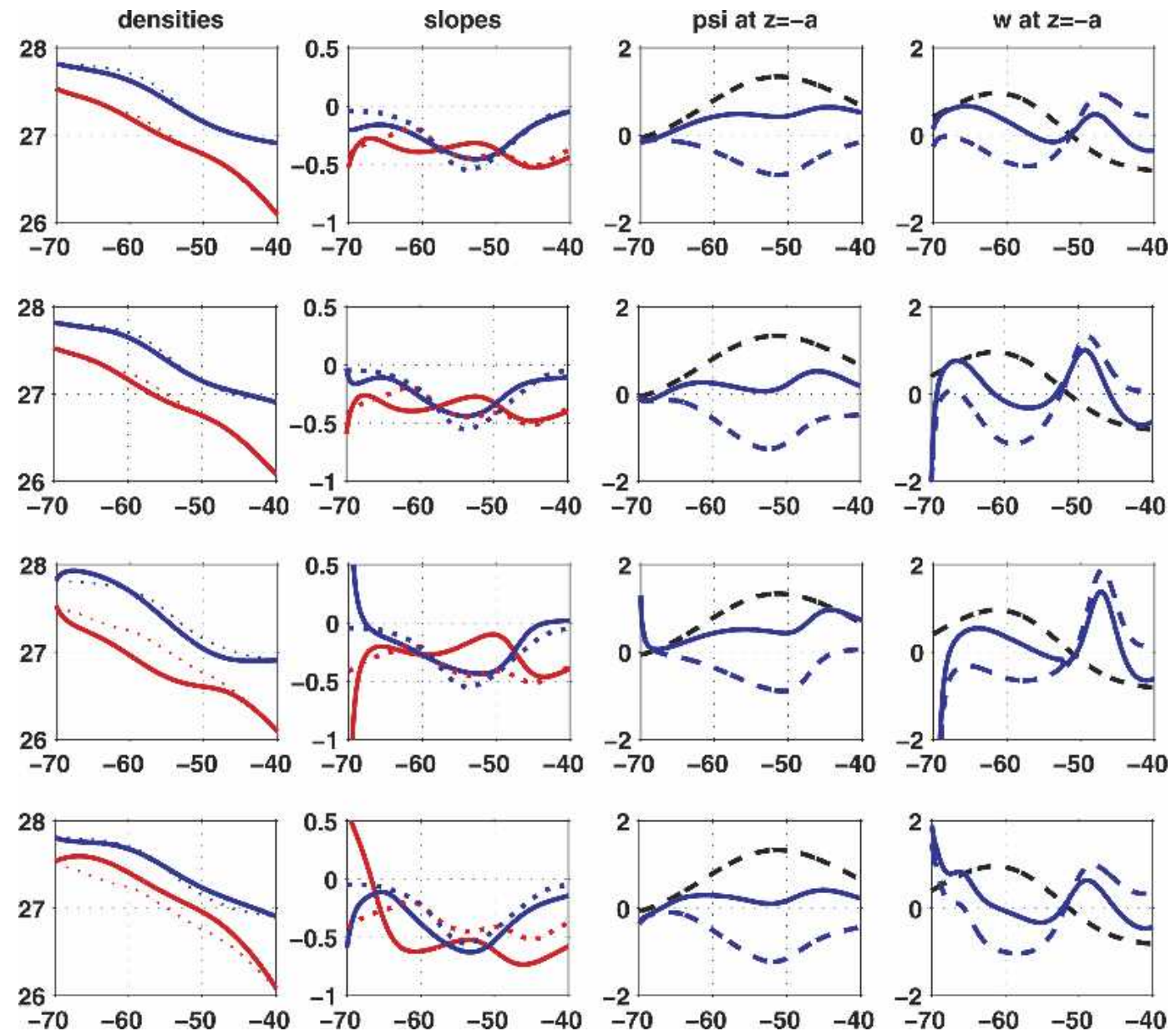

FIG. 9. Solutions for alternative parameter choices and complete forcing functions: (first row) $K_{i}=1500 \mathrm{~m}^{2} \mathrm{~s}^{-1}$; (second row) $K_{s}=750 \mathrm{~m}^{2} \mathrm{~s}^{-1}$; (third row) $\alpha_{d}=2.81 \times 10^{-7} \mathrm{~m} \mathrm{~s}^{-1}, \alpha_{a}=10^{-6} \mathrm{~m} \mathrm{~s}^{-1}$; and (fourth row) $\alpha_{d}=1.69$ $\times 10^{-6} \mathrm{~m} \mathrm{~s}^{-1}, \alpha_{a}=6 \times 10^{-6} \mathrm{~m} \mathrm{~s}^{-1}$. All other parameters and curve notation are as in Fig. 8 .

the forcing in a rather nonlocal way: it relates to the meridional density gradient $B_{a}^{\prime}$ on the interface and the spreading $d y^{\dagger} / d y$ of the isopycnals, both introducing nonlocal relations to the forcing $\mathcal{M}$ and $\mathcal{H}_{0}$. Notice that the mapping transfers the meridional scale of the density field in the upper layer into a vertical scale of the current profile. Likewise, vertical integrals can be mapped into meridional integrals along the interface $z$ $=-a$. Hence integrating (19) twice we find for the transport (relative to the bottom):

$$
\begin{aligned}
\mathcal{T} & =\int_{-h}^{-a} U(y, z) d z \\
& =\frac{g}{f} \int_{y s}^{y} \frac{Z\left(y, y^{\dagger}\right)+a}{K(y)}\left[K^{\dagger} s^{\dagger}+\mathcal{M}^{\dagger}-\mathcal{M}(y)\right] B_{a}^{\prime}\left(y^{\dagger}\right) d y^{\dagger},
\end{aligned}
$$

where $Z\left(y, y^{\dagger}\right)$ is the isopycnal depth at $y$ of the iso- pycnal starting at $y^{\dagger}$ [see the appendix; note that we have assumed a vertically constant eddy diffusivity, $K(y, z)=K(y)$, in the interior]. The dagger quantities are taken at $y^{\dagger}$. We thus have two contributions to the transport: one relating to the local wind stress $\tau_{0}(y)=$ $-f \mathcal{M}(y)$, which roughly scales as $-g \Delta B \tau_{0} h_{m} /\left(K f^{2}\right)$, and one relating to the residual streamfunction $\psi_{\text {res }}^{\dagger}=K^{\dagger} s^{\dagger}$ $+\mathcal{M}^{\dagger}$ at the interface, which scales as $-g \Delta B h_{m} \psi_{\mathrm{res}}^{\dagger}{ }^{\prime}$ $(K f)$. Both are proportional to the buoyancy difference $-g \Delta B$ across the Antarctic zone and a mean isopycnal depth $h_{m}$. We may thus write the transport as

$$
\mathcal{T} \sim \frac{-g \Delta B h_{m}}{K f^{2}}\left(\tau_{0}+f \psi_{\mathrm{res}}^{\dagger}\right)
$$

The residual circulation term in this relation is neglected in Marshall and Radko (2003). However, it is generally not small (see, e.g., in the simulations shown in Figs. 8 and 9). Notice that for a northward-decreasing 

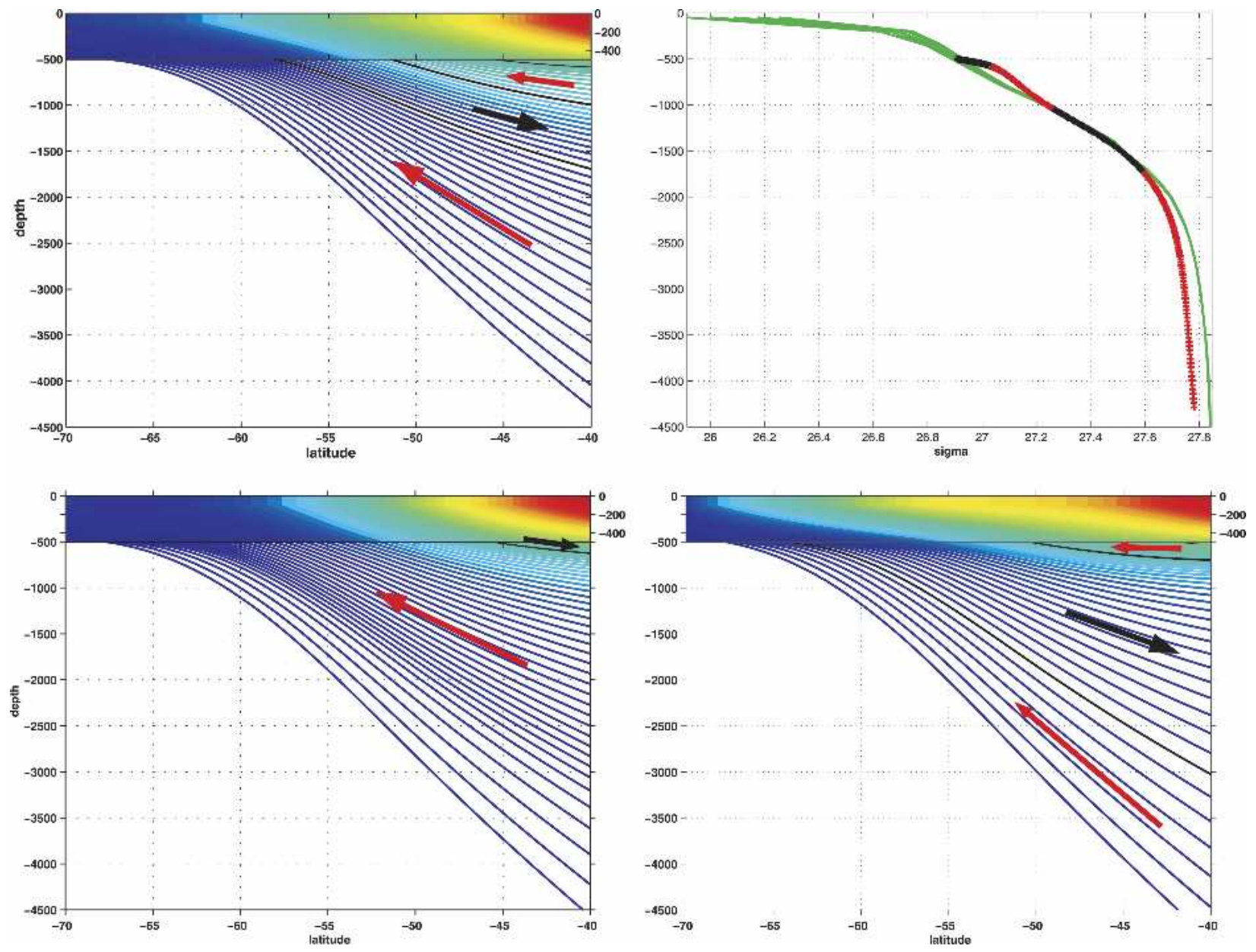

FIg. 10. The density field for the upper-layer simulations of Fig. 8 . The eddy diffusivity in the interior is $K_{i}=500 \mathrm{~m}^{2} \mathrm{~s}^{-1}$ at the southern boundary, linearly increasing by a factor of 5 toward the northern side and vertically constant. For the upper $500 \mathrm{~m}$ the solutions $B_{d}$ and $B_{a}$ have been converted to contours; below, the characteristics are colored according to the corresponding density. At black characteristics upward residual transport (red arrows) changes to downward (black arrow). (top) Wind stress and density flux forcing. (top right) The resulting density values on the northern boundary at $-40^{\circ}$ (red and black curve; red is upwelling and black is downwelling) in comparison with the SAC densities (between $-42^{\circ}$ and $-38^{\circ}$; green profiles). The density field for the cases of (bottom left) zero density flux and (bottom right) zero wind stress.

density $(\Delta \mathrm{B}<0)$ the wind term yields an eastward transport. The sign of the other contribution is not unique but it is clearly negative for our standard set of parameters (see Fig. 8) and the cases with parameters variations (see Fig. 9). The eddies do not overcome the Ekman part in these solutions and the part forced by the local wind stress would stand as an upper limit of the transport. A more complete scaling requires relating these delicate dependencies as well as $\Delta \mathrm{B}$ and $h_{m}$ on the forcing parameters.

\section{Summary and conclusions}

The isopycnals in the Southern Ocean connect the deep ocean to the north of the ACC to the surface areas to the south, the ACC being attached to stronger tilts correlated over the depth. We have turned existing concepts (e.g., Marshall 1997; Speer et al. 2000; Marshall and Radko 2003) on processes shaping the isopycnal stack in the Southern Ocean into a prognostic theory. For these processes we refer to Fig. 1. The Eulerian mean flow and the eddies combine to transport density (heat and substances) to and from the upper-ocean layer, where mixing by small-scale turbulence and exchange of heat and freshwater with the atmosphere must occur. Two assumptions imply that the mean transport in the interior-below the mixed layer-by the mean flow and eddies is entirely along the isopycnals. These are 1) eddies do not carry material across isopycnals, only along them, and 2) diapycnal mixing by 
small-scale turbulence is neglected below the mixed layer. Then streamlines of transport by mean flow and eddies, representing the residual circulation, coincide with isopycnals. The concept allows one to break the complicated mathematics of an advection-diffusionmixing regime into manageable parts-mixed layer physics with Ekman and eddy advection and an adiabatic interior- which may be solved by simpler means. We are able to predict the density field - the decrease of surface density with increasing latitude and the shape of the downward sloping isopycnals-from the wind field and buoyancy flux through the ocean surface.

The theory and resulting solutions demonstrate the overwhelming importance of the transient eddy field in shaping the isopycnals in the Southern Ocean. The eddy-induced vertical pumping at the interface of the upper layer to the interior largely compensates the Ekman pumping (as the Deacon cell is masked by eddyinduced transports; see Döös and Webb 1994). It should also be remembered that the meridional eddy transport of density (or heat) leads to the interfacial eddy form stress that governs with its vertical divergence the zonal balance of momentum of the ACC (see, e.g., Rintoul et al. 2001; Olbers et al. 2004). The interior density field is as well strongly dependent on eddy effects. In the present theory the isopycnal slopes and-via characteristics propagation-the thermocline depth and, finally, the baroclinic zonal transport of the ACC are structured by the magnitude and pattern of the eddy diffusivity $K$. It is clear that a larger $K$ in the interior leads to a shallower thermocline and a smaller ACC transport.

We have avoided using parameterizations of $K$ (as the one used, e.g., by Visbeck et al. 1997; Marshall and Radko 2003), not because they might be questionable but rather not to diversify or mask the basic concepts too much. Instead, we present results from a simple inverse study that relates observed isopycnal slopes and net water mass transports to the magnitude and shape of eddy diffusivity profiles. We find that eddy mixing coefficients are pretty tightly constrained to be between 800 and $1200 \mathrm{~m}^{2} \mathrm{~s}^{-1}$ below $500-\mathrm{m}$ depth with a significant increase toward upper-ocean values between 2000 and $4000 \mathrm{~m}^{2} \mathrm{~s}^{-1}$. This expected increase in (adiabatic) eddy mixing will most likely be accompanied by significant diabatic mixing that our theory takes into account.

A second major topic is turbulent mixing. The real ocean is diabatic with mixing across isopycnals by small-scale turbulence, but it is still in debate if it occurs predominantly between the outcropping isopycnals in the surface layer (as we assume for simplicity in the present study) or in the interior as well. Only if there is exchange of mass between the isopycnal layers south of the respective latitude, implying conversion of water masses south of the ACC can the meridional overturning transports be nonzero: they equal the net exchange rate with the neighboring layers (integral of diapycnal transport divergence over the area south of the respective latitude). At the same time, the overturning transports imply a Coriolis force in the individual isopycnal layers that is in balance with nonzero vertical divergence of the interfacial form stress. Eddy effects at the respective latitude and diabatic interior effects of smallscale turbulence occurring to the south must thus adjust according to mass and momentum requirements of the zonal current and the meridional overturning.

There are a few inconsistencies in the model, which we discuss in the following. The main objection comes probably from the handling of boundary conditions. Repeating the model philosophy, we have constructed a solution for the quasi-streamline-averaged density field from the forcing by density flux and wind stress at the sea surface with reasonable and simple parameterizations of eddies and SGS mixing. An important aspect, however, are the boundary conditions of the density balance in the ocean interior, which in our model is purely advective with velocities associated with the residual streamfunction. Evidently, in such a situation we should implement advective boundary conditions, that is, a prescription of density values at inflow points and no prescription at outflow points. In the present setup we thus should prescribe the density at the upper-layer base where pumping is downward (this is established) and at the northern boundary where advection is southward. The latter condition (occurring mainly at the NADW inflow) is not implemented. It would require the knowledge of the inflow range while integrating the upper-layer balances and thus would result in a completely coupled upper layer-interior ocean model concept and thus lead to some iterative integration procedure of our upper-layer model and the interior characteristic problem. Instead, in the present state of the model, we prescribe densities also for the inflow range at the upper-layer base [in form of the "deep" density profile $\tilde{B}(y)$ representing density at about $600-800 \mathrm{~m}]$. In view of the good reproduction of the AAIW density structure in the present simulation, we might be relieved, but the conceptual inconsistency is surely present.

Another drawback appears in the incomplete treatment of the Ekman overturning streamfunction. For simplicity we have disregarded the deep-topography and bottom form stress based-closure of the streamfunction, as sketched in Fig. 2. It would introduce another arbitrariness of a not well-known structure func- 
tion (of the bottom form stress) and presumably would put the model beyond its limits.

The treatment of mixing is extremely simple. The upper two layers in the model have constant depths and are defined such that they span all of the turbulent mixing and this is done by the constant mixing parameters $\alpha_{d}$ and $\alpha_{a}$ placed at the layer interfaces. One may envision more aspects of mixed layer physics with a predictive module on the mixed layer depth and the mixing efficiency. We might also wish to implement a more interactive atmosphere that reacts on the upward pumped water and creates the surface fluxes (at least of heat) instead of prescribing them entirely.

Further refinement of the model would more affect the model's simplicity. We consider the above drawbacks as of secondary importance and ignore them in our solutions. They could in principle be resolved and more refined parameterization be included. But even in the present state the model has its value as a simple tool to investigate the dependence of stratification and overturning in the Southern Ocean on surface fluxes, eddy effects, and turbulent mixing.

Acknowledgments. We appreciate numerous discussions with Richard Greatbatch, John Marshall, Timour Radko, Stephen Rintoul, and Sergey Danilov. Support of DO by LDEO and CSIRO during extended visits is gratefully acknowledged.

\section{APPENDIX}

\section{Solution of the Characteristic Equation}

The characteristic problem (9) results in $d z / d y=s$, $d K s / d y=-\mathcal{M}^{\prime}$ with solution [taking for simplicity $T(z)$ $\equiv 1$ and a vertically constant $K=K(y)]$

$$
\begin{aligned}
K(y) s(y, z)+\mathscr{M}(y)= & K^{\dagger} s^{\dagger} \mathcal{M}^{\dagger} \\
z=Z\left(y, y^{\dagger}\right)= & -a+\int_{y^{\dagger}}^{y}\left[K^{\dagger} s^{\dagger}+\mathcal{M}^{\dagger}-\mathcal{M}\left(y^{\prime}\right)\right] \\
& \times \frac{d y^{\prime}}{K\left(y^{\prime}\right)},
\end{aligned}
$$

where the daggered quantities refer to the footprint $y=$ $y^{\dagger}$ of the characteristic (isopycnal) on the interface level $z=-a$. Two isopycnals that start at $y^{\dagger}$ and $y^{\dagger}+d y^{\dagger}$ on $z=-a$ will hence be separated by $d y$ on the level $z$, given by the relation

$$
\begin{aligned}
& {\left[K^{\dagger} s^{\dagger}+\mathcal{M}^{\dagger}-\mathcal{M}(y)\right] \frac{d y}{K(y)}=} \\
& \quad\left[s^{\dagger}-\frac{\partial}{\partial y^{\dagger}}\left(K^{\dagger} s^{\dagger}+\mathcal{M}^{\dagger}\right) \int_{y^{\dagger}}^{y} \frac{d y^{\prime}}{K\left(y^{\prime}\right)}\right] d y^{\dagger} .
\end{aligned}
$$

Zero spreading, $d y=d y^{\dagger}$, occurs if there is no Ekman pumping (i.e., $\mathcal{M}=$ const) and $K=$ const and the slope on the interface is constant (i.e., $s^{\dagger}=$ const).

\section{REFERENCES}

Andrews, D. G., and M. E. McIntyre, 1976: Planetary waves in horizontal and vertical shear: The generalized Eliassen-Palm relation and the mean zonal acceleration. J. Atmos. Sci., 33, 2031-2048.

— Drazin theorems for waves on axisymmetric mean flows in compressible atmosphere. J. Atmos. Sci., 35, 175-185.

—, J. R. Holton, and C. B. Leovy, 1987: Middle Atmosphere Dynamics. International Geophysics Series, Vol. 40, Academic Press, 489 pp.

Cunningham, S. A., S. G. Alderson, B. A. King, and M. A. Brandon, 2003: Transport and variability of the Antarctic Circumpolar Current in Drake Passage. J. Geophys. Res., 108, 8084, doi:10.1029/2001JC001147.

Döös, K., and D. J. Webb, 1994: The Deacon cell and other meridional cells of the Southern Ocean. J. Phys. Oceanogr., 24, 429-442.

Fritzsch, B., R. Gerdes, W. Hiller, M. Latif, S. Legutke, E. MaierReimer, D. Olbers, and F. Roeske, 2000: Vergleich der thermohalinen Zirkulation in zwei globalen ozeanischen Zirkulationsmodellen-The Ocean Model Intercomparison Project. Abschlussbericht BMBF Projekt 01 LA 9862/8, 133 pp.

Gent, P. R., and J. C. McWilliams, 1990: Isopycnal mixing in ocean circulation models. J. Phys. Oceanogr., 20, 150-155.

Green, J. S. A., 1970: Transfer properties of the large-scale eddies and the general circulation of the atmosphere. Quart. J. Roy. Meteor. Soc., 96, 157-185.

Held, I. M., and T. Schneider, 1999: The surface branch of the zonally averaged mass transport circulation in the troposphere. J. Atmos. Sci., 56, 1688-1697.

Heywood, K., A. C. N. Garabato, and D. P. Stevens, 2002: High mixing rates in the abyssal Southern Ocean. Nature, 415, 1011-1014.

Marshall, D., 1997: Subduction of water masses in an eddying ocean. J. Mar. Res., 55, 201-222.

Marshall, J., and T. Radko, 2003: Residual-mean solutions for the Antarctic Circumpolar Current and its associated overturning circulation. J. Phys. Oceanogr., 33, 2341-2354.

McIntosh, P. C., and T. J. McDougall, 1996: Isopycnic averaging and the residual mean circulation. J. Phys. Oceanogr., 26, $1656-1660$

Naveira Garabato, A. C., K. L. Polzin, B. A. King, K. J. Heywood, and M. Visbeck, 2004: Widespread intense turbulent mixing in the Southern Ocean. Science, 303, 210-213.

Olbers, D., and V. O. Ivchenko, 2001: On the meridional circulation and balance of momentum in the Southern Ocean of POP. Ocean Dyn., 52, 79-93.

— D. D. Borowski, C. Völker, and J.-O. Wolff, 2004: The dynamical balance, transport and circulation of the Antarctic Circumpolar Current. Antarct. Sci., 16 (4), 79-110.

Orsi, A. H., S. S. Jacobs, A. L. Gordon, and M. Visbeck, 2001: Cooling and ventilating the abyssal ocean. Geophys. Res. Lett., 28, 2023-2026.

— W. M. Smethie, and J. L. Bullister, 2002: On the total input of Antarctic waters to the deep ocean: A preliminary esti- 
mate from chlorofluorocarbon measurements. J. Geophys. Res., 107, 3122, doi:10.1029/2001JC000976.

Phillips, H. E., and S. R. Rintoul, 2000: Eddy variability and energetics from direct current measurements in the Antarctic Circumpolar Current south of Australia. J. Phys. Oceanogr., 30, 3050-3076.

Rintoul, S. R., and S. Sokolov, 2001: Baroclinic transport variability of the Antarctic Circumpolar Current south of Australia (WOCE repeat section SR3). J. Geophys. Res., 106, 28152832.

, C. Hughes, and D. Olbers, 2001: The Antarctic Circumpolar Current system. Ocean Circulation and Climate, G. Siedler, J. Church, and J. Gould, Eds., Academic Press, 271-302.

Schmitz, W. J., Jr., 1995: On the interbasin-scale thermohaline circulation. Rev. Geophys., 33, 151-173.

_ 1996a: On the world ocean circulation: Volume I. Some global features/North Atlantic circulation. Tech. Rep. WHOI-96-03, Woods Hole Oceanographic Institution, Woods Hole, MA, 140 pp.

, 1996b: On the world ocean circulation: Volume II. The Pa- cific and Indian Oceans/A global update. Tech. Rep. WHOI96-08, Woods Hole Oceanographic Institution, Woods Hole, MA, 237 pp.

Sloyan, B. M., and S. R. Rintoul, 2001: Circulation, renewal, and modification of Antarctic mode and intermediate waters. $J$. Phys. Oceanogr., 31, 1005-1030.

Speer, K., S. Rintoul, and B. Sloyan, 2000: The diabatic Deacon cell. J. Phys. Oceanogr., 30, 3212-3222.

Stone, P. H., 1972: A simplified radiative-dynamical model for the static stability of rotating atmospheres. J. Atmos. Sci., 29, 405-418.

Sverdrup, H. U., M. W. Johnson, and R. H. Fleming, 1942: The Oceans: Their Physics, Chemistry and General Biology. Prentice-Hall, 1087 pp.

Trenberth, K. E., and J. M. Caron, 2001: Estimates of meridional atmosphere and ocean heat transports. J. Climate, 14, 3433 3443.

Visbeck, M., J. Marshall, T. Haine, and M. Spall, 1997: Specification of eddy transfer coefficients in coarse-resolution ocean circulation models. J. Phys. Oceanogr., 27, 381-402. 